\section{P4.17 CONTRACEPTIVE PREFERENCE AMONG FEMALE SEX WORKERS IN THE SIVET STUDY IN NAIROBI, KENYA}

Borna Nyaoke. Kavi-Institute of Clinical Research, University of Nairobi, Nairobi, Kenya

10.1136/sextrans-2017-053264.514

Introduction Long-acting reversible contraceptives (LARCs) are effective birth control methods that do not depend on patient compliance. They include injections, intrauterine devices (IUDs) and sub-dermal implants. Kenya had over 1.5 million people living with HIV in 2015 with female sex workers (FSWs) known to be at a higher risk for both unintended pregnancies and HIV infection. HIV clinical trials test novel compounds whose effects in fetuses are unknown thus require female subjects to use LARCs to avoid pregnancies. Clinical trials also aim to recruit high-risk individuals to closely study infection dynamics in a representative natural infection cohort. Therefore, it is crucial to investigate the preference for contraception in a HIV-high risk population such as FSWs to inform the design and conduct of HIV vaccine clinical trials, especially in the resource-limited Sub-Saharan Africa. The on-going Simulated Vaccine Efficacy Trial (SiVET) Study at KAVI-ICR may provide answers to this.

Methods Data on use of modern contraception methods (pills, injectables, implants, IUDs and surgicals) are collected from FSWs during screening, and confirmed by a contraceptive card or the presence of an implant or IUD strings. The proportion of women per method used is determined.

Results Seventy nine women with an age range of 18-50 years have been screened since 2016. Most (89\%, $n=73)$ were already using contraception. Of these; $63 \%(n=46)$ were on injectables, $22 \%$ on implants $(n=16), 8 \%$ IUCDs $(n=6), 4 \%$ pills $(n=3)$ and $3 \%(n=2)$ had undergone a surgical method. No pregnancy while two HIV infections were reported at screening.

Conclusion There is high contraceptive use among the FSWs with the majority preferring injectables. This practice is encouraging and thus, discontinuation of volunteers from the clinical trial due to pregnancy is unlikely. At the end of trial in 2018, data will be collected on changes in contraceptive, number of pregnancies, condom use, HIV infections and experience on use of the contraception.

\section{P4.18 NOT SO NORMALISED - PATIENT PERSPECTIVES ON HIV DIAGNOSIS AND TREATMENT DECISIONS: RESULTS FROM A LARGE QUALITATIVE STUDY IN LONDON}

${ }^{1} \mathrm{PJ}$ Bruton, ${ }^{1} \mathrm{~T}$ Rai, ${ }^{1} \mathrm{SE}$ Day, ${ }^{2} \mathrm{C}$ Higgs, ${ }^{2} \mathrm{~J}$ Rowlands, ${ }^{1}$ Helen Ward. ${ }^{1}$ Imperial College London, London, UK; ${ }^{2}$ Chelsea and Westminster NHS Foundation Trust, London, UK

\subsection{6/sextrans-2017-053264.515}

Introduction Improvements in treatment and life expectancy have led to HIV being increasingly regarded as "just another chronic disease". We hypothesised that the experience of being diagnosed and starting treatment for HIV would have changed over the decades of the epidemic to reflect this normalisation. Methods Qualitative interview study of 52 people attending two large HIV clinics in London, purposively sampled to include people diagnosed in "generations" from 1980s to 2015, analysed using framework approach.

Results Despite the improvements in antiretroviral therapy (ART) and life expectancy over the last 3 decades, the experience of receiving a diagnosis of HIV remained strikingly similar across the generations, being one of shock and fear of death, recalling thinking that "this is the end" or that " $m y$ expiry date was stamped on me now". This did not vary with level of knowledge or experience of having previous HIV tests. In contrast, the decision to start ART was more complex in the early days when potential gains had to be weighed against side effects and depended on clinical monitoring. For those diagnosed in the 1980s and 90s, it was often seen as a defeat "I fought it all this time on my own, and then finally I had to give in and take a pill. That was kind of depressing". But in the last decade the decision although easier in some ways was still a major event. People across the generations reported being worried about accidental disclosure through taking medications, fear of the short and long term side effects, and described how taking daily medications became a constant reminder of their HIV status; one participant described starting medication as "worse than being diagnosed". Conclusion HIV may appear as "just another chronic disease" to some clinicians, but for people being diagnosed and invited to start treatment these are major life events, as they are for many chronic conditions. To ensure good linkage to and retention in care, clinicians should acknowledge this and facilitate appropriate support.

\section{P4.19 FACTORS ASSOCIATED WITH STI DIAGNOSIS AND TESTING AMONG MEN WHO HAVE SEX WITH MEN IN JAMAICA}

${ }^{1}$ Carmen Logie, ${ }^{2}$ Kathleen Kenny, ${ }^{3}$ Peter A Newman, ${ }^{4}$ Kandasi Levermore, ${ }^{5}$ Nicolette Jones, ${ }^{4}$ Ava Neil, Tyrone Ellis4; ${ }^{6}$ Annecka Marshall. 'University of Toronto, Toronto, Canada, Canada; ' University of North Carolina, Chapel Hill, Chapel Hill, Us, Canada; ${ }^{3}$ University of Toronto, Toronto, Canada; ${ }^{4}$ Jamaica AIDS Support for Life, Kingston - Jamaica; ${ }^{5}$ Jamaica AIDS Support for Life, Kingston, Canada; ${ }^{6}$ University of West Indies, Mona Campus, Kingston - Jamaica

\subsection{6/sextrans-2017-053264.516}

Introduction Little is known about factors associated with STI acquisition and testing among men who have sex with men (MSM) in Jamaica despite reported high HIV prevalence. The study objective was to examine correlates of lifetime STI history and testing among MSM in Jamaica.

Methods We conducted a cross-sectional tablet-based survey with MSM recruited using peer-driven sampling methods in Kingston, Ocho Rios, and Montego Bay, Jamaica. We estimated factors associated with ever having an STI test and lifetime history of STI. Bivariable regression assessed the strength of associations between potential factors with outcomes of interest. Logit-link models were used to estimate final multivariable models and fit using backwards-stepwise regression.

Results Among 556 participants, 67 (14\%) were HIV-positive and $416(75 \%)$ reported a previous STI test. In the final multivariable model, STI testing history was associated with sociodemographic (graduated high school [OR: 2.34, 95\% CI: 1.30 , 4.20]) and community (perceived sexual stigma [OR: 1.10, 95\% CI: 1.03, 1.16]; lesbian, gay, and bisexual community connectedness [OR: 1.12, 95\% CI: 1.01, 1.23]) factors. Participants who were casually dating had lower odds of STI testing than those in a relationship (OR: $0.56,95 \%$ CI: $0.34,0.94$ ). Among participants who had received a STI test and result $(n=404), 49(12 \%)$ reported a lifetime STI history. In the final multivariable model, lifetime STI history was associated with increased odds of HIV seropositivity (OR: 3.88; 95\% CI $1.80,8.35$ ), current unemployment (OR: 2.24, 95\% CI: 1.12 , 
4.46), and inconsistent condom use (OR: 2.23; 95\% CI: 1.05 , 4.75).

Conclusion MSM in Jamaica report high prevalence of STI and HIV. STI testing rates are suboptimal and associated with complex multi-level (socio-demographic, relationship, community) factors. Lifetime history of STI was associated with HIV co-infection, structural factors (unemployment), and sexual practices (inconsistent condom use). Results can inform multilevel STI prevention and care programs tailored for MSM in Jamaica.

\section{P4.20 FACTORS ASSOCIATED WITH OPTING IN FOR CLINIC- BASED SYPHILIS TESTING AMONG TRANSGENDER WOMEN IN JAMAICA}

${ }^{1}$ Carmen Logie, ${ }^{2}$ Kathleen Kenny, ${ }^{3}$ Peter A Newman, ${ }^{4}$ Kandasi Levermore, ${ }^{5}$ Nicolette Jones, ${ }^{4}$ Ava Neil, ${ }^{4}$ Tyrone Ellis, ${ }^{6}$ Annecka Marshall. ${ }^{1}$ University of Toronto, Toronto, Canada, Canada; ${ }^{2}$ University of North Carolina, Chapel Hill, Chapel Hill, US; ${ }^{3}$ University of Toronto, Toronto, Canada; ${ }^{4}$ Jamaica AIDS Support for Life, Kingston - Jamaica; ${ }^{5}$ Jamaica AIDS Support for Life, Kingston, Canada; ${ }^{6}$ University of West Indies, Mona Campus, Kingston Jamaica

\subsection{6/sextrans-2017-053264.517}

Introduction Transgender women experience high sexually transmitted infection (STI) rates, yet there is a lack of information about STI testing uptake among transgender women in low and middle-income countries. We conducted a tablet-based survey to assess syphilis testing uptake and prevalence among transgender women in Jamaica.

Methods We conducted a cross-sectional survey with a peerdriven recruitment sample of transgender women in Kingston and Ocho Rios, Jamaica. Participants were provided with a coupon with their survey identification (ID) code for voluntary, free, rapid serological syphilis testing. Coupon ID codes for testing uptake/results were linked with survey results. We conducted backwards stepwise logistic regression to determine factors associated with opting in for syphilis testing.

Results Among 137 participants (mean age: 24.0 [SD: 4.5]), $60.6 \%$ opted in for syphilis testing and $10.6 \%$ tested positive. One-quarter (25.2\%) self-reported being HIV-positive; all participants with syphilis infection were HIV-positive. In univariable analyses having multiple partners was associated with reduced odds of opting in for testing (OR: 0.19; 95\% CI: 0.06-0.60). In multivariable analyses controlling for relationship status, HIV-positive participants were four-fold more likely to opt-in for syphilis testing (Adjusted Odds Ratio [AOR: 4.33]; 95\% CI: 1.31-14.26) than HIV-negative participants. Perceived STI risk (AOR: 1.58; 95\% CI: 1.04-2.40) and childhood sexual abuse history (AOR: 2.80; 95\% CI: 1.03-7.62) were associated with increased odds of opting in for testing. Incarceration history (AOR: 0.27; 95\% CI: 0.110.71 ) was associated with reduced odds of opting in for syphilis testing.

Conclusion Transgender women in Jamaica experience high HIV and syphilis prevalence, and syphilis and HIV co-infection. Findings suggest opt-in clinic based syphilis testing may miss the opportunity to provide testing for some transgender women at elevated STI risk. Future research should assess whether point-of-care syphilis testing may increase testing uptake.

\section{P4.21 SIDE CHICKS, THOTS AND SIDE DICKS: TYPOLOGIES AND DRIVERS OF CONCURRENT PARTNERSHIPS AMONG PEOPLE OF BLACK CARIBBEAN ETHNICITY IN ENGLAND AND IMPLICATIONS FOR STI PREVENTION AND PARTNER NOTIFICATION}

Cath Mercer, ${ }^{1,2} \mathrm{~S}$ Wayal, ${ }^{1,2}$ Gilbart VI, 1,2 E Garnett, ${ }^{1,2}$ Sutcliff Lj, 1,3 P Weatherburn, ${ }^{1,4} \mathrm{G}$ Hughes. 'National Institute of Health Research (NIHR) Health Protection Research Unit (HPRU) in Blood Borne and Sexually Transmitted Infections, UK; ${ }^{2}$ Centre for Sexual Health and HIV, Research Department of Infection and Population Health, University College London, UK; 3 London School of Hygiene and Tropical Medicine, London, UK; ${ }^{4}$ Public Health UK

\subsection{6/sextrans-2017-053264.518}

Introduction In Britain, STI diagnoses rates are highest among black Caribbeans compared to other ethnic groups. The prevalence of concurrency is also high in this population. Concurrent partnerships, (i.e. having sexual partners overlapping in time), can enhance the rate and speed of STI transmission. We explored typologies and drivers of concurrency in black Caribbeans in England and considered their implications for STI prevention.

Methods Using purposive sampling, we recruited people of black Caribbean ethnicity aged $\geq 15$ years from community settings and STI clinics. Audio-recorded 4 focus group discussions ( $\mathrm{n}=28$ participants) and 24 in-depth interviews were conducted between June 2014-Dec 2015 using topic guides. Data was transcribed, managed using NVivo software and analysed using thematic framework to identify patterns of concurrent partnerships and condom use, and reasons for concurrency.

Results 32 women and 20 men (age range: 15-70 years) identifying as heterosexual participated. Open, situational, and experimental concurrent partnerships were commonly reported. Open concurrent partnerships involved a person having a main sexual partner and, in the case of men, additionally having sex with other "side chicks" or "thots", and in the case of women, "side dicks". Situational partnerships involved having a sexual relationship with an ex-partner, especially with someone with whom they had had a child, while having another main sexual partner. These types of partnerships were usually long-term, and condoms were less likely to be used due to emotional attachment, if a co-parent was single and then condomless sex was perceived as a way to "entice" the partner back, or due to the relationship being founded on sexual pleasure. Usuallypeople were aware of the concurrent nature of these partnerships. Experimental concurrent partnerships, commonly reported by single participants, were usually short term, and mostly involved condom use. These were fuelled by lack of readiness to settle with a single partner, or trying to figure out the type of partner they may want to settle with. Other commonly reported reasons for concurrency were low self-esteem at an individual level. At a socio-cultural level, although concurrency was frowned upon, it was perceived as "a Black Caribbean thing" with references being 
commonly made in certain types of popular music and dance among black Caribbeans, changing norms of relationships in an era where you can "order sex via app", peer pressure, and a normalisation of concurrency on social media, especially among men.

Conclusion Among black Caribbeans, the different types and contexts of concurrent partnerships can have implications for STI prevention. Awareness of being in a concurrent partnership could potentially facilitate uptake of interventions including condom use, partner notification, and reduce the risk of re-infection. In addition, such interventions should address broader sociocultural factors influencing risk behaviour including the impact of media.

\section{P4.22 CROSSING THE BRIDGE: EXPLORING SEXUAL RISK PROFILES OF MEN WHO HAVE SEX WITH MEN ATTENDING A SEX ON PREMISES VENUE AND A PUBLIC SEXUAL HEALTH CLINIC FOR STI SCREENING IN SYDNEY, AUSTRALIA}

Catriona Ooi, David Lewis. Western Sydney Sexual Health Centre, Parramatta, Australia

10.1136/sextrans-2017-053264.519

Introduction In Australia, men who have sex with men (MSM) are targeted to curb the spread of HIV and STIs. 'Non-gay' identifying MSM (NGMSM) may not identify with health messages, impacting knowledge of HIV/STI harm minimisation and result in poorer health-seeking behaviours. NGMSM and other men who have sex with men and women are often hidden to clinical services. These men may act as bridges for HIV/STI transmission to female partners. Novel strategies to reach this group include opportunistic HIV/STI screening at sex on premises venues (SOPV). We compared the demographics, testing and sexual behaviour of MSM attending a SOPV to MSM attending an established sexual health clinic (SHC).

Method A daytime SOPV HIV/STI screening service was conducted 2-3 consecutive days per month from November 2015 for 12 months. All patrons were offered testing. The comparison group were MSM attending a local SHC for screening the week following each SOPV clinic. The SHC operates weekdays with appointment and walk-in options. At both sites, participants consented to provide demographic information, contact details and a brief sexual history. Demographics, sexual behaviour and testing practices were compared between the 2 groups.

Results During the study period 84 men tested at the SOPV and 108 at the SHC. SOPV testers were older (mean age, 48.4 years. vs 34.6 years.; $\mathrm{p}<0.001$ ) and were more likely to have had sex with a female in the past 12 months (49/84, $58 \%$ vs. $19 / 105,18 \% ; \mathrm{p}<0.001)$. Compared with SHC testers, more SOPV testers had never had an HIV test $(23 / 84$, $27 \%$ vs. $12 / 108,11 \% ; \mathrm{p}<0.01)$. In the previous 3 months, $100 \%$ condom use with regular partners was similar in both groups (SOPV 33/84, 39\% vs. SHC 37/105, 35\%; p=0.67).

Conclusion Inconsistent condom use with casual and regular sex partners, combined with higher reported rates of sex with females, may enable SOPV testers to act as bridges for STI/ HIV transmission between MSM and heterosexual populations. Our findings have implications for HIV/STI service provision, contact tracing and local health promotion initiatives.
P4.23 EGOCENTRIC SEXUAL NETWORK CHARACTERISTICS OF MEN WHO HAVE SEX WITH MEN (MSM) AND TRANSGENDER WOMEN (TW) RECENTLY DIAGNOSED WITH SYPHILIS AND/OR GONORRHOEA/CHLAMYDIA (GC/CT) IN LIMA, PERU: NETWORK PATTERNS AS ROADMAPS FOR STI PREVENTION INTERVENTIONS

${ }^{1}$ Cherie S Blair, ${ }^{1}$ Eddy R Segura, ${ }^{2}$ Amaya Perez-Brumer, ${ }^{3}$ Jorge Sanchez, ${ }^{4}$ Javier R Lama, ${ }^{1}$ Jesse L Clark. 'David Geffen School of Medicine At UCLA, Department of Medicine and Program in Global Health, Los Angeles, USA; ${ }^{2}$ Columbia University Mailman School of Public Health, New York, USA; ${ }^{3}$ Universidad Nacional Mayor San Marcos, Lima - Peru; ${ }^{4}$ Asociación Civil Impacta Salud y Educación, Lima - Peru

\subsection{6/sextrans-2017-053264.520}

Introduction Endemic rates of STIs like GC/CT and syphilis in Peru are only partially explained by individual behavioural or biological factors. Characterisation of sexual networks of MSM/TW with syphilis and/or GC/CT can provide critical data to inform prevention efforts.

Methods We enrolled $917 \mathrm{MSM} / \mathrm{TW}$ as screening for 2 STI control trials in Lima, Peru. We surveyed demographics and sexual identity, role, and 30 day network characteristics (number/gender of sexual partners, partner types, and frequency of anal/vaginal intercourse) and tested for syphilis (RPR >1:16) and oral, rectal, or urethral GC/CT (TMA). Differences in egocentric network characteristics were analysed with Chisquare and Kruskal-Wallis tests.

Results Approximately 38.7\% $(\mathrm{n}=355)$ of subjects had a new STI diagnosis (Syphilis: 97 [10.6\%]; GC/CT: 161 [17.6\%]; Syphilis-GC/CT Co-infection: 49 [5.3\%]). MSM/TW with GC/ CT were younger (median age+IQR: 25 [22-30]) than those with syphilis (28 [23-34]) or no STI (28 [24-35]). STI-negative subjects were more likely to identify their sexual role as activo (insertive; $24.8 \%$ ) than men with syphilis $(10.8 \%)$ or GC/CT (14.7\%). MSM/TW with GC/CT reported greater median numbers of all partners (3 [2-5]) and of casual male or transgender partners $(2[0-4])$ than those with syphilis $(2[1-5]$ and 1 [0-2], respectively) or no STI (2 [1-3] and 1 [0-2]). Both $\mathrm{GC} / \mathrm{CT}$ and syphilis were associated with the number of partnerships involving receptive anal intercourse (RAI; 2 [1-4] and 2 [1-13]) compared with STI-uninfected subjects (1 [0-2]). No differences were noted in the number of partnerships with condomless RAI or number of female partners.

Conclusion Egocentric network characteristics of MSM/TW with GC/CT and/or syphilis demonstrated progressive increases in network size, number of casual partners, and frequency of RAI when comparing no infection vs. syphilis vs. GC/CT +/syphilis co-infection. Detailed understanding of network patterns, along with individual and partnership characteristics, will inform public health responses to HIV/STIs among Latin American MS.

\section{P4.24 SWIPE LEFT ON DISEASE: THE EFFECTS OF CONTEXTUAL FACTORS AND DATING APP USAGE ON SEXUAL RISK BEHAVIOURSAMONG YOUNG ADULTS}

Chloe Chan. New York University, New York, USA

\subsection{6/sextrans-2017-053264.521}

Introduction With the rise of social media and smartphones, millennials increasingly report meeting their romantic partners through mobile dating apps. Yet there is concern that this may also be the reason behind increases in sexually 